\title{
Flies get into renewal
}

How tissues might replenish themselves after injury has fascinated biologists for decades; however, research in this field has frequently been hampered by the lack of an easy-to-use experimental model. Now, a paper reports a means of studying regeneration in arguably the genetically most tractable organism of all - Drosophila melanogaster - thereby opening up the phenomenon to genetic dissection on an unprecedented scale.

Much recent progress in understanding regeneration has come from knockdown screens in planaria or mutagenesis screens in zebrafish. Even so, there is an incentive to develop approaches that do not rely on inflicting surgical lesions, which are time consuming and laborious.
The fruit fly contains imaginal discs - small pouches of cells in the larva that develop into adult appendages such as wings and legs - that are known to regenerate after injury or transplantation, but technical issues have confounded the study of their regeneration. Smith-Bolton and colleagues have developed a simple way of damaging a portion of the wing disc in a way that the extent of regeneration can easily be inferred from the size of the adult wing. They first induced spatially and temporally controlled lesions, then characterized the cellular and molecular mechanisms behind tissue regeneration, and finally showed that this system can be used for systematic functional studies.

Tissue damage was induced by placing an apoptosis-promoting gene, either eiger (the fly tumour necrosis factor- $\alpha$ orthologue) or reaper (another inducer of apoptosis), under the control of a promoter that drives expression in the wing pouch (see the image) and then using a temperature shift to switch expression on and then off. Having defined the developmental time during which regeneration occurs (before the middle of the final larval stage), the authors characterized the response to injury. For example, they found that cells proliferate around the site of injury but proliferation is reduced elsewhere, and that regenerated tissue derives from both eigerexpressing cells and the surrounding non-expressing cells. Functional studies were then carried out to determine whether the genes and mechanisms that operate following injury in the fly are similar to those that function in other organisms. In particular, wingless expression, which is involved in regeneration in other systems, is delocalized and expressed at the highest levels in the proliferating cells of the pouch; gain-of-function and loss-offunction studies revealed that this wingless expression inhibits Notch activity, which, in turn, allows Myc expression and hence regeneration. Overexpressing Myc - but not other growth regulators, such as Cyclin D - hastens the natural recovery process, although the effect is only seen in the developmental window that is permissive for regeneration.

Perhaps the most important aspect of this paper is the demonstration that the disc ablation system is a suitable platform for the unbiased identification of other molecular components, through the use of genetic screens. As a proof of principle, the authors used a series of deletion lines for one chromosome arm to identify regions that, when hemizygous, modified adult wing size after ablation. The genes underlying these effects are yet to be characterized.

The new fly system is a controllable means of ablating cells in vivo that, importantly, does not require surgical intervention. The system should be readily transferable to other imaginal discs, which will hopefully provide a fruitful basis for large-scale screens.

Tanita Casci

ORIGINAL RESEARCH PAPER Smith-Bolton, R. K., Worley, M. I., Kanda, H. \& Hariharan, I. K. Regenerative growth in Drosophila imaginal discs is regulated by Wingless and Myc. Dev. Cell 16, 797-809 (2009) FURTHER READING Sánchez Alvarado, A. \& Tsonis, P. A. Bridging the regeneration gap: genetic insights from diverse animal models. Nature Rev Genet. 7, 873-884 (2006) 